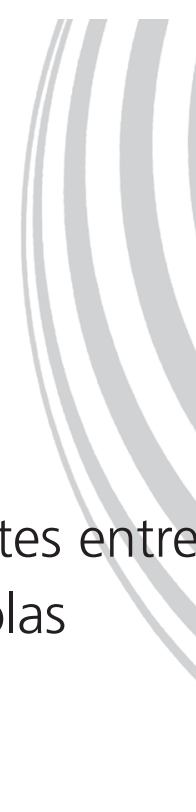

ORIGINAL | ORIGINAL

\title{
Consumo alimentar de micronutrientes entre pré-escolares no domicílio e em escolas de educação infantil do município de Caxias do Sul (RS)
}

\author{
Dietary micronutrient intake of preschool children \\ at home and in kindergartens of the municipality \\ of Caxias do Sul (RS), Brazil
}

\author{
Juliana Rombaldi BERNARDI ${ }^{1}$ \\ Carla De CEZARO' \\ Regina Mara FISBERG² \\ Mauro FISBERG ${ }^{3}$ \\ Guilherme Pádua RODRIGUES 4 \\ Márcia Regina VITOLO'
}

RE S U M O

\section{Objetivo}

Avaliar a ingestão alimentar de micronutrientes em pré-escolares no domicílio e em escolas de educação infantil públicas e particulares.

\section{Métodos}

Estudo transversal realizado com 362 pré-escolares entre dois e seis anos de idade, em Caxias do Sul (RS) Brasil, em 2007. A ingestão alimentar na escola foi avaliada por meio do método de pesagem direta individual, e no domicílio, por meio de registro alimentar realizado pelos pais ou responsáveis. Foi calculada a ingestão alimentar de cálcio, ferro, folato, vitamina A, vitamina C e zinco de acordo com o local da refeição e tipo de escola.

\section{Resultados}

Houve maior ingestão de alimentos contendo ferro, folato e vitamina $C$ durante o período em que as crianças permaneceram na escola infantil, e maior ingestão de cálcio, vitamina A e zinco no domicílio. Houve

\footnotetext{
1 Universidade Federal de Ciências da Saúde de Porto Alegre, Programa de Pós-Graduação em Ciências da Saúde. R. Sarmento Leite, 245, 90050-170, Porto Alegre, RS, Brasil. Correspondência para/Correspondence to: J.R. BERNARDI. E-mail: $<$ juliana.bernardi@yahoo.com.br>.

2 Universidade de São Paulo, Faculdade de Saúde Pública, Departamento de Nutrição. São Paulo, SP, Brasil.

3 Universidade Federal de São Paulo, Departamento de Pediatria. São Paulo, SP, Brasil.

${ }^{4}$ Coordenador de Assuntos Científicos, Danone Research. Palaiseau Cedex, França.
} 
254 | J.R. BERNARDI et al.

significativamente maior ingestão de alimentos contendo ferro $(p=0,03)$, folato $(p=0,03)$, vitamina $A(p<0,01)$ e vitamina $C(p<0,01)$ pelas crianças da escola particular e maior ingestão de cálcio $(p<0,01)$ e zinco $(p<0,01)$ na escola pública. Quanto à prevalência de inadequação dos micronutrientes, as crianças não apresentaram risco deficiente para ingestão de ferro, folato, vitamina A e C e zinco, porém apenas 67,4\% apresentaram ingestão de cálcio igual ou acima ao valor de referência.

\section{Conclusão}

Os achados sugerem que o consumo de cálcio, vitamina A e zinco foi maior nos domicílios, apesar de as crianças permanecerem a maior parte do dia nas escolas. O consumo diário de micronutrientes de crianças de escolas públicas e particulares não diferiu significativamente, mesmo com diferenças nos cardápios.

Termos de indexação: Consumo de alimentos. Creches. Micronutrientes. Pré-escolar.

\section{A B S T R A C T}

\section{Objective}

This study assessed the adequacy of preschool children's dietary micronutrient intake at home and in public and private kindergartens.

\section{Methods}

This cross-sectional study done in 2007 included 362 preschool children aged 2-6 years from Caxias do Sul (RS), Brazil. Food intake at school was assessed by the individual direct weighing method and at home by food records kept by the parents or caregivers. Dietary intakes of calcium, iron, folate, vitamin A, vitamin C and zinc were calculated according to meal location and type of school.

\section{Results}

Dietary iron, folate and vitamin C intakes were higher in kindergartens and dietary intakes of calcium, vitamin $A$ and zinc were higher at home. Dietary intakes of iron $(p=0.03)$, folate $(p=0.03)$, vitamin $A(p<0.01)$ and vitamin $C(p<0.01)$ were significantly higher in private kindergartens and dietary intakes of calcium $(p<0.01)$ and zinc $(p<0.01)$ were significantly higher in public kindergartens. Intakes of iron, folate, vitamin $A$, vitamin $C$ and zinc were adequate but only $67.4 \%$ of the children were ingesting enough calcium.

\section{Conclusion}

Dietary intakes of calcium, vitamin A and zinc were higher at home even though the children remained at school during most of the day. The daily micronutrient intakes of children from private and public kindergartens were not significantly different despite the menu differences.

Indexing terms: Food consumption. Child day care centers. Micronutrients. Child, preschool

\section{N T R O D U Ç Ã O}

As deficiências alimentares podem levar ao crescimento e ao desenvolvimento inadequado das crianças, aumentando sua vulnerabilidade às infecções e gerando atrasos no processo de maturação do sistema nervoso e no desenvolvimento mental e intelectual, podendo ser irreversíveis dependendo da intensidade e da duração da deficiência ${ }^{1,2}$.

Quando o consumo de energia e de nutrientes está abaixo das necessidades, estabelecem-se as condições para o aparecimento das doenças carenciais, sendo a anemia ferropriva, a deficiência de vitamina $A$ e a desnutrição as mais comuns na faixa pré-escolar ${ }^{3,4}$. Segundo a Pesquisa Nacional de Demografia e Saúde (PNDS) ${ }^{5}$, houve prevalência de anemia em 20,9\% de 3.455 crianças até cinco anos e níveis inadequados de vitamina A de 17,4\% em 3.499 crianças da mesma faixa etária.

Além disso, o consumo alimentar das crianças é influenciado por condicionantes socioeconômicos da família, como renda e escolaridade dos pais, e pelo conhecimento e cuidado da mãe ou responsável ao acesso, seleção e aquisição dos alimentos. Porém, é importante evitar 
que as mudanças nos padrões dietéticos não propiciem o aparecimento de hábitos alimentares incorretos, independente do estrato socioeconômico da família ${ }^{6}$.

Como existe atualmente proporção relevante e crescente de crianças em idade pré-escolar em serviços institucionalizados públicos e particulares ${ }^{7,8}$, e como há escassez de estudos representativos com crianças que frequentam escolas de educação infantil ${ }^{9}$, pesquisas que avaliem a ingestão alimentar de pré-escolares nesse ambiente são necessários.

O objetivo deste estudo foi avaliar a ingestão alimentar de cálcio, ferro, folato, vitamina $\mathrm{A}$, vitamina $C$ e zinco de crianças entre dois a seis anos de idade em escolas infantil públicas e particulares e nos domicílios.

\section{M É T O D O S}

Estudo transversal realizado no município de Caxias do Sul (RS), integrante de projeto maior, multicêntrico, intitulado: "Nutri Brasil Infância"10, com o objetivo principal de avaliar a prevalência de inadequação da ingestão de nutrientes nas crianças brasileiras, realizado nas cidades de Manaus, Natal, Recife, Brasília, Cuiabá, Rio de Janeiro, Belo Horizonte e São Paulo.

Os dados foram coletados durante o ano de 2007 por duas nutricionistas e dez estudantes de graduação em nutrição, em escolas de educação infantil públicas e particulares. Participaram do estudo crianças de ambos os sexos entre dois e seis anos de idade, que permaneciam preferencialmente em período integral em instituições que ofereciam a refeição do almoço e que possuíam serviço de distribuição convencional das refeições, isto é, porcionadas pela funcionária responsável em servir a alimentação. Assim, foram selecionadas as três maiores escolas de educação infantil pública da cidade e seis escolas particulares que aceitaram participar do estudo e que preenchiam os critérios elegíveis de seleção. As escolas participantes localizavam-se tanto na região central quanto na periferia da cidade.
O número amostral estipulado foi de 350 crianças: 250 frequentadoras de escolas de educação infantil públicas e 100 de particulares, constituindo-se amostra de conveniência dos pesquisadores responsáveis.

Para a classificação segundo a recomendação de micronutrientes, as crianças foram separadas em dois grupos, de acordo com a faixa etária, compreendendo o grupo 1 (G1) crianças entre dois a três anos de idade, e o grupo 2 (G2), crianças entre quatro e seis anos de idade. Foram excluídas do estudo crianças enfermas no dia da coleta de dados. Para a determinação do perfil econômico das crianças analisadas foi aplicado aos pais o questionário da Associação Brasileira de Empresas de Pesquisas ${ }^{11}$.

\section{Avaliação da ingestão alimentar}

Todas as crianças tiveram um dia de seu consumo alimentar avaliado, e, para a obtenção da variabilidade intraindividual, foram sorteados aleatoriamente $25 \%$ das crianças para participarem de uma subamostra em que tiveram mais um dia de consumo avaliado, com pelo menos um dia de intervalo entre as coletas.

Os alimentos e as preparações consumidas nas escolas de educação infantil foram avaliados pelo Método de Pesagem Direta Individual (MPDI), refletindo o que a criança comeu apenas no período em que se encontrava na escola, isto é, em parte da manhã até o período da tarde, sendo as refeições oferecidas: lanche da manhã, almoço, lanche da tarde e pré-janta. Os alimentos consumidos em casa foram avaliados por informações fornecidas pelos pais ou responsáveis por meio do registro alimentar, refletindo o que a criança comeu no período em que não estava na escola, isto é, em parte da manhã e no período da noite.

Na escola de educação infantil, realizou-se três vezes a pesagem de todos os alimentos oferecidos às crianças e calculou-se a média de cada alimento. Realizadas as refeições, foi quantificado o resto alimentar de cada criança e descon- 
tado da média de cada alimento realizada anteriormente.

A ingestão alimentar da casa foi registrada pelos pais ou pelos responsáveis, correspondendo ao mesmo dia em que foi realizada a pesagem na escola de educação infantil. Os registros foram submetidos a análises detalhadas quanto ao conteúdo nutricional e devidamente esclarecidas questões dele provenientes. O registro alimentar consistiu no preenchimento, em formulários especialmente desenhados, dos alimentos e bebidas consumidos ao longo do período solicitado, incluindo os alimentos consumidos fora do lar.

Com o objetivo de otimizar e facilitar a organização dos dias de coleta dos dados, os dias de pesagem de alimentos na escolas infantis foram intercalados com os dias de recolhimento dos registros alimentares preenchidos pelos pais.

O cálculo do valor nutritivo dos alimentos consumidos e registrados foi realizado utilizando-se o programa Nutrition Data System (NDS, version 2007, Nutrition Coordinating Center [NCC], University of Minnesota, Minneapolis).

A estimativa da prevalência de inadequação de ferro, folato e vitaminas A e C foi calculada pelo método da necessidade média estimada (EAR - Estimated Average Requirement) como ponto de corte, considerando-se em risco a proporção (superior a 15\%) de crianças do grupo que não alcançaram os valores de referência estabelecidos para o nutriente. Nos nutrientes cuja EAR ainda não está estabelecida, como o cálcio, não foi possível estimar a prevalência de inadequação, sendo utilizados como referência os valores da ingestão adequada (Al - Adequate Intake), para tanto avaliou-se a proporção de crianças com ingestão igual ou superior ao valor de referência ${ }^{12-14}$.

A análise dos dados foi realizada utilizando o programa estatístico Statistical Package for Social Science (SPSS), versão 11.0. Foram descritas as frequências simples e percentuais da distribuição da idade, do sexo e da classe econômica das crianças. Com base na replicação da medida dietética, foram calculados os parâmetros necessários para a estimativa da ingestão habitual. 0 método desenvolvido por Nusser et al. ${ }^{15}$ foi utilizado para o cálculo dos componentes de variância e para sua aplicação na estimativa da ingestão habitual de nutrientes.

Descreveram-se as Médias (M) e o Desvio-Padrão (DP) da ingestão alimentar de cálcio, ferro, folato, vitamina A, vitamina $\mathrm{C}$ e zinco, de acordo com os locais das refeições realizadas pelas crianças (escola ou domićlio) e o tipo de escola (pública ou particular). As comparações da ingestão alimentar entre as escolas foram realizadas por meio do teste não-paramétrico Mann-Whitney $\mathrm{U}$, indicado pelo teste de Kolmogorov-Smirnov, pois todos os micronutrientes analisados mostraram-se de distribuição assimétrica. Considerou-se nível de significância de 5,0\% $(p \leq 0,05)$.

Este projeto foi aprovado pelo Comitê de Ética em Pesquisa da Universidade Federal de Ciências da Saúde de Porto Alegre (UFCSPA) com o número de aprovação 532/07-CEP e no Comitê de Ética em Pesquisa do Hospital de São Paulo com o número 0617/07.

Foram obtidos os Termos de Consentimento Livre e Esclarecido de todos os responsáveis pelas crianças para participar do estudo.

\section{RESULTADOS}

Este estudo investigou a ingestão alimentar de micronutrientes de 362 pré-escolares, de ambos os sexos, $28,7 \%(n=104)$ provenientes de escolas de educação infantil particulares e 71,3\% ( $n=258$ ) de escolas públicas em Caxias do Sul (RS). A média de idade das crianças foi de aproximadamente quatro anos ( $M=4,29, D P=1,13)$, e $46 \%$ da amostra eram do gênero masculino.

Em relação à distribuição da classe econômica, aproximadamente 83\% (87/104) das crianças das escolas infantis particulares pertenciam às classes econômicas mais altas, como a $A 1(n=1)$, A2 $(n=28), B 1(n=37)$ e B2 $(n=21)$. Em contrapartida, aproximadamente $83 \%$ (216/258) das crian- 
ças frequentadoras das escolas infantis públicas pertenceram às classes econômicas mais baixas, como a $C(n=170)$ e $D(n=46)$. Não houve criança da escola infantil pública pertencente à classe econômica $A 1$, e apenas duas crianças $(1,9 \%)$ da escola infantil particular pertenceram à classe $D$. Além disso, não houve no estudo criança representante da classe econômica $\mathrm{E}$.

Conforme distribuição etária, o grupo 1 (G1), crianças entre dois a três anos, representou $39,2 \%$ ( $n=142$ ) da amostra, e o grupo 2 (G2), crianças entre quatro a seis anos, representou $60,8 \%(n=220)$. Foi avaliada a média de ingestão alimentar dos micronutrientes cálcio, ferro, folato, vitamina $A$, vitamina $C$ e zinco em ambos os grupos.

Pode-se observar (Tabela 1) que houve consumo maior de alimentos contendo ferro, folato e vitamina $C$ em ambos os grupos etários durante o período em que as crianças estiveram na escola infantil. Houve, em contrapartida, maior ingestão de alimentos contendo cálcio, vitamina A e zinco durante o período em que as crianças estiveram no domicílio. Assim, calculando a proporção de cada nutriente nos ambientes escolar e domiciliar em relação à ingestão alimentar total diária e unindo os dois grupos etários (G1 e G2), foi encontrado que $56,5 \%$ da ingestão alimentar de ferro do dia das crianças foram fornecidos pela escola de educação infantil, conjuntamente com $60,5 \%$ da ingestão de folato e $59,4 \%$ da ingestão de vitamina C. Em contrapartida, $62,7 \%$ da ingestão diária de cálcio, 55,3\% da ingestão de vitamina $A$ e $51,4 \%$ da ingestão de zinco foram fornecidos pelo domićlio.

Análise complementar no domicílio, por meio da avaliação dos registros alimentares, mostrou que a maior parte das crianças, $86,8 \%$ ( $n=317)$, consumiu laticínios: leite, achocolatados e açúcar ou farinhas, iogurte, queijo, doce de leite e nata.

Os dados apresentados na Tabela 2 mostram que no período das escolas houve significativamente maior consumo de alimentos contendo ferro $(p=0,03)$, folato $(p=0,03)$, vitamina $A$ $(p<0,01)$ e vitamina $C(p<0,01)$ pelas crianças de escolas particulares, em comparação às de escolas públicas. No entanto, as crianças de escolas públicas tiveram significativamente maior ingestão de alimentos contendo cálcio $(p<0,01)$ e zinco $(p<0,01)$, em comparação às de escolas particulares.

Tabela 1. Ingestão alimentar diária de micronutrientes entre crianças de 2 a 3 anos e entre crianças de 4 a 6 anos. Caxias do Sul (RS), 2007.

\begin{tabular}{|c|c|c|c|c|c|c|}
\hline \multirow{2}{*}{ Nutrientes } & \multicolumn{2}{|c|}{ Domicílio } & \multicolumn{2}{|c|}{ Escola infantil } & \multicolumn{2}{|c|}{ Consumo total } \\
\hline & M & DP & M & DP & M & DP \\
\hline \multicolumn{7}{|l|}{ Grupo G1 $(n=142)$} \\
\hline Cálcio (mg) & 546,09 & 243,06 & 306,49 & 105,17 & 852,58 & 246,86 \\
\hline Ferro (mg) & 4,61 & 2,25 & 5,90 & 2,25 & 10,52 & 3,17 \\
\hline Folato (mg) & 148,92 & 59,73 & 217,26 & 70,42 & 366,19 & 93,28 \\
\hline Vitamina A (mg) & 268,78 & 147,18 & 213,41 & 26,50 & 482,20 & 150,92 \\
\hline Vitamina C (mg) & 31,64 & 25,79 & 44,66 & 15,09 & 76,30 & 28,55 \\
\hline Zinco (mg) & 4,40 & 1,75 & 4,01 & 1,79 & 8,41 & 2,33 \\
\hline \multicolumn{7}{|l|}{ Grupo G2 $(n=220)$} \\
\hline Cálcio (mg) & 499,48 & 251,91 & 308,90 & 109,16 & 808,39 & 250,80 \\
\hline Ferro (mg) & 4,95 & 2,08 & 6,52 & 1,89 & 11,47 & 2,96 \\
\hline Folato (mg) & 160,18 & 63,14 & 252,37 & 69,22 & 412,55 & 93,19 \\
\hline Vitamina A (mg) & 258,08 & 208,22 & 210,94 & 23,79 & 469,03 & 208,91 \\
\hline Vitamina C (mg) & 30,33 & 23,05 & 45,44 & 15,06 & 75,77 & 28,16 \\
\hline Zinco (mg) & 4,65 & 2,26 & 4,47 & 1,49 & 9,12 & 2,66 \\
\hline
\end{tabular}

M: média; DP: desvio-padrão. 
258 | J.R. BERNARDI et al.

Tabela 2. Comparação da ingestão alimentar de micronutrientes das crianças nas diferentes escolas. Caxias do Sul (RS), 2007.

\begin{tabular}{|c|c|c|c|c|c|}
\hline \multirow{2}{*}{ Nutrientes } & \multicolumn{2}{|c|}{ Escola pública } & \multicolumn{2}{|c|}{ Escola particular } & \multirow{2}{*}{$p^{*}$} \\
\hline & $M$ & DP & $\mathrm{M}$ & DP & \\
\hline Cálcio (mg) & 321,23 & 102,88 & 275,02 & 111,97 & $<0,01^{\text {** }}$ \\
\hline Ferro (mg) & 6,18 & 2,05 & 6,50 & 2,08 & $0,03^{* *}$ \\
\hline Folato (mg) & 233,91 & 64,86 & 250,23 & 85,55 & $0,03^{* *}$ \\
\hline Vitamina A (mg) & 208,60 & 21,98 & 220,14 & 29,47 & $<0,01^{* *}$ \\
\hline Vitamina C (mg) & 42,80 & 11,88 & 50,91 & 19.88 & $<0,01^{* *}$ \\
\hline Zinco (mg) & 4,45 & 1,65 & 3,89 & 1,51 & $<0,01^{* *}$ \\
\hline
\end{tabular}

* Valor de $p$ segundo teste não-paramétrico Mann-Whitney $U$, indicado pelo teste de Kolmogorov-Smirnov; ${ }^{\star *}$ valor de $p$ significativo, $p \leq 0,05$. M: média; DP: desvio-padrão.

Com relação à prevalência de inadequação dos micronutrientes analisados, as crianças não apresentaram risco para ingestão deficiente de ferro, folato, vitamina A e C e zinco. Das crianças frequentadoras de escolas privadas e públicas, $66,3 \%(n=69 / 104)$ e $67,8 \%(n=175 / 258)$, respectivamente, apresentaram ingestão de cálcio igual ou acima do valor de referência.

\section{I S C U S S Ã O}

Este estudo original compara a ingestão alimentar de micronutrientes entre pré-escolares de escolas de educação infantil públicas e particulares, e aplica a estimativa da ingestão habitual com replicação de dados utilizando as abordagens recomendadas pelo Institute of Medicine ${ }^{16}$ e pela Organização Mundial de Saúde ${ }^{17}$.

Os estudos já publicados com crianças institucionalizadas realizaram avaliação nutricional ${ }^{18}$ ou analisaram carências de micronutrientes específicos (anemia, hipovitaminose A) das crianças frequentadoras de escolas infantis ${ }^{8,9,19-21}$ ou avaliaram os serviços institucionalizados de acordo com algumas normas técnicas de infraestrutura e de práticas de higiene ${ }^{7}$. Outros estudos, de igual importância, também avaliaram a ingestão de crianças pré-escolares frequentadoras de escolas infantis, analisando o consumo diário total de alguns micronutrientes ${ }^{21,22}$.

No presente trabalho, por meio da análise complementar dos registros alimentares, a maior quantidade de alimentos contendo cálcio, vita- mina A e zinco no período do domicílio pode ser explicada pela maior ingestão de produtos lácteos das crianças nesse ambiente. Farias Júnior \& Osório $^{2}$, em estudo realizado em Pernambuco com 969 crianças menores de cinco anos, encontraram que o leite, dentre os alimentos analisados, foi consumido por maior número de crianças $(86,8 \%)$.

A proporção de ingestão de zinco foi considerada a mais semelhante entre os ambientes domiciliar e escolar $(51,4 \%$ vs. $48,5 \%$ respectivamente). Dentre as fontes alimentares de zinco, pode-se sugerir que a maior proporção de sua ingestão na casa seja também provinda da quantidade de fontes lácteas consumidas, porém sua ingestão na escola deve-se provavelmente às fontes de alta biodisponibilidade, como as carnes.

A ingestão alimentar de ferro, folato e vitamina $C$, superior no ambiente escolar, pode ser explicada pelo fato de a escola infantil oferecer as principais refeições do dia23, como o almoço e a pré-janta, cuja composição têm importância tanto do ponto de vista dietético quanto nutritivo, com maior ingestão pelas crianças de carne e vegetais, importantes fontes de ferro, folato e zinco. Além disso, foi observada a oferta de frutas como sobremesa nas refeições principais, contribuindo na ingestão alimentar de vitamina $C$ diária das crianças. Porém, embora as refeições oferecidas fossem as mesmas para todas as crianças, seu consumo é variável devido às preferências individuais ou em função das refeições repetidas pelas crianças. 
Em relação ao ambiente das escolas infantis, semelhante aos achados neste estudo, pesquisa realizada por Barbosa et al. ${ }^{24}$ confirma a proteção que o serviço institucionalizado proporciona às crianças, pois foi observada melhora significativa no consumo de micronutrientes importantes como ferro (de 5,1 mg para 7,8mg) e vitamina C (de 31,5mg para 81,3mg), comparando-os nos dois momentos do estudo, no ato da matrícula e após seis meses de frequência na escola infantil. Outro estudo transversal realizado por Silva et al. ${ }^{19} \mathrm{em}$ São Paulo, comparando crianças atendidas em escolas infantis e aquelas não-atendidas pertencentes à mesma comunidade, verificou que a escola infantil foi considerada fator de proteção para o estado nutricional. Assim, programas que tenham por base os cuidados infantis disponibilizados de forma integral com qualidade adequada, destinados às crianças, podem exercer papel preventivo no sentido de protegê-las de distúrbios nutricionais, principalmente em populações menos favorecidas. $\mathrm{O}$ acesso à creche e o acompanhamento das crianças, portanto, devem ser assegurados.

Nos dias atuais, as escolas de educação infantil estão se tornando uma necessidade na população em consequência das transformações socioeconômicas ocorridas nas últimas décadas. Como as crianças permanecem na escola de oito a dez horas por dia e, durante esse tempo, recebem dois terços de suas necessidades nutricionais, além da orientação psicopedagógica, é necessário que a alimentação e os cuidados oferecidos satisfaçam suas necessidades e influenciem favoravelmente seu estado nutricional e seu desenvolvimento neuropsicomotor ${ }^{25}$. Diante disso, cada vez mais, a escola é considerada um espaço estratégico para incentivo à formação de hábitos alimentares saudáveis ${ }^{26}$.

Encontramos neste estudo que, no período em que as crianças permaneceram nas escolas particulares, consumiram mais alimentos contendo ferro, folato, vitamina A e vitamina C em comparação às crianças das escolas públicas, que consumiram mais alimentos contendo cálcio e zinco.
Isso sugere a necessidade de melhoria nos cardápios e alimentos oferecidos nas instituições públicas, já que na escola são realizadas as principais refeições (almoço e pré-janta), que devem, consequentemente, suprir maior proporção de micronutrientes na alimentação diária da criança. Porém, a escolha do tipo da escola de educação infantil muitas vezes não acontece com base em princípios e critérios da família, pois geralmente nas escolas públicas há insuficiência de vagas diante da demanda e nas escolas particulares há a questão do elevado custo do serviço ${ }^{27}$.

Apesar de o consumo dos micronutrientes no ambiente escolar ser diferente entre o período da escola pública e particular, as crianças não apresentaram risco na ingestão de ferro, folato, vitamina $\mathrm{A}$ e $\mathrm{C}$ e zinco e houve similaridade da prevalência de adequação de cálcio entre as crianças das diferentes escolas. Esses resultados demonstram que, apesar das diferenças dos cardápios oferecidos pelas escolas infantis, a ingestão de micronutrientes acaba sendo alcançada no domicílio. Assim, as crianças frequentadoras de escolas particulares, que recebem maiores fontes de ferro, folato, vitamina $A$ e vitamina $C$ nesse período, recebem maior quantidade de cálcio e zinco em seus domicílios. Já as crianças frequentadoras de escolas públicas, que recebem maiores quantidades de cálcio e zinco nesse período, provavelmente ingerem maior quantidade de ferro, folato, vitamina A e vitamina C em casa.

Para que as crianças, independente de suas condições socioeconômicas, recebam uma alimentação adequada, com os nutrientes necessários para seu desenvolvimento, é necessária política efetiva que garanta seu acesso às escolas infantis e que essas sejam agentes promotores da educação nutricional infantil. Além disso, implementação de ações para melhorar a qualidade das refeições oferecidas pelas escolas, como padronização de cardápios, adequado gerenciamento de orçamento na compra dos alimentos, educação alimentar para as crianças e funcionários, são necessários ${ }^{28}$. Porém, como o tempo de permanência da criança na escola infantil não é 
260 | J.R. BERNARDI et al.

suficiente para o atendimento total das recomendações nutricionais, faz-se necessário que a família assuma parte dessa responsabilidade por meio de hábitos alimentares adequados, não somente para a criança, mas também no âmbito familiar domiciliar24,29,30.

\section{O N C L U S Ã O}

Em síntese, este trabalho demonstrou que o consumo, entre as crianças, de cálcio, vitamina A e zinco foi maior no domicílio, mesmo permanecendo a maior parte do dia na escola. Apesar das diferenças de cardápios das escolas, o consumo diário de micronutrientes de crianças de escolas públicas e particulares não diferiu significativamente.

Os achados sugerem que estratégias e ações de caráter preventivo sejam planejados e implementados no ambiente dos pré-escolares, principalmente na adequação do cardápio das escolas públicas. É fundamental a garantia de acesso das crianças aos serviços institucionalizados para acompanhar e assegurar condições adequadas de crescimento e de desenvolvimento. Além disso, as escolas devem estabelecer estratégias de educação alimentar aos pais para orientá-los quanto aos alimentos que devem ser oferecidos às crianças no período que permanecem nos domicílios.

\section{OLABORADORES}

J.R. BERNARDI e C. De CEZARO responsável pela coleta de dados, análise dos dados, elaboração do rascunho e da revisão crítica do conteúdo e participação da aprovação da versão final do manuscrito. R.M. FISBERG responsável pela elaboração do protocolo e análise dos dados e participação da aprovação da versão final do manuscrito. M. FISBERG coordenador do projeto, elaboração do protocolo e análise dos dados. G.P. RODRIGUES elaboração do protocolo e análise dos dados. M.R. VITOLO responsável pela análise dos dados, elaboração do rascunho, da revisão crítica do conteúdo e participação da aprovação da versão final do manuscrito.

\section{REFERÊ NCIAS}

1. Cruz GF, Santos RS, Carvalho CM, Moita GC. Avaliação dietética em creches municipais de Teresina, Piauí, Brasil. Rev Nutr. 2001; 14(1):21-32. doi: 10.15 90/S1415-52732001000100004.

2. Farias Júnior G, Osório MM. Padrão alimentar de crianças menores de cinco anos. Rev Nutr. 2005; 18(6):793-802. doi: 10.1590/\$1415-52732005000 600010.

3. Falcão-Gomes RC, Coelho AAS, Schimitz BAS. Caracterização dos estudos de avaliação do consumo alimentar de pré-escolares. Rev Nutr. 2006; 19(6): 713-27. doi: 10.1590/S1415-527320060006000 08.

4. Fidelis CMF, Osório MM. Consumo alimentar de macro e micronutrientes de crianças menores de cinco anos no Estado de Pernambuco, Brasil. Rev Bras Saude Matern Infant. 2007; 7(1):63-74. doi: 10.1590/S1519-38292007000100008.

5. Programa Nacional de Demografia e Saúde da Criança e da Mulher. Anemia e hipovitaminose A. Brasil, 2009. Disponível em: <http:// www.saude. gov.br/pnds2006>.

6. Aquino RC, Philippi ST. Consumo infantil de alimentos industrializados e renda familiar na cidade de São Paulo. Rev Saúde Pública. 2002; 36(6): 655-60. doi: 10.1590/S0034-891020020007000 01.

7. Barros AJD, Halpern R, Menegon OE. Creches públicas e privadas de Pelotas, RS: aderência à norma técnica. J Pediatr (Rio de Janeiro). 1998; 74(5): 397-403.

8. Bueno MB, Marchiori DML, Fisberg RM. Evolução nutricional em crianças atendidas em creches públicas no Município de São Paulo, Brasil. Rev Panam Salud Publica. 2003; 14(3):165-70. doi: 10.1590/S1020-49892003000800003.

9. Bueno MB, Selen SSD, Arêas JAG, Fisberg RM. Prevalência e fatores associados à anemia entre crianças atendidas em creches públicas de São Paulo. Rev Bras Epidemiol. 2006; 9(4):462-70. doi: 10.1590/S1415-790X2006000400007.

10. Fisberg M, Fisberg RM, Maximino P, Bueno M, Rodrigues GP. Brazilian National Survey of food intake and nutritional status of children 2-5 years - Nutri Brasil Infancia Project. Ann Nutr Metabol. 2009; 55(Suppl.1):340-54. doi: 10.1159/000248293.

11. Associação Brasileira de Empresas de Pesquisa. Dados com base no levantamento sócio-econômico 2000. Brasil, 2003. Disponível em: <http:// www.abep.org $>$.

12. United States of American. Institute of Medicine. National research council dietary reference intakes 
for vitamin A, vitamin K, arsenic, boron, chromium, copper, iodine, iron, manganese, molybdenum, nickel, silicon, vanadium and zinc. Washington (DC): National Academic Press; 2002.

13. United States of American. Institute of Medicine. Dietary reference intakes for calcium, phosphorus, magnesium, vitamin D, and fluoride. Washington, (DC): National Academy Press; 1999.

14. United States of American. Institute of Medicine. Dietary reference intakes for thiamin, riboflavin, niacin, vitamin B6, folate, vitamin B12, pantothenic acid, biotin, and choline. Washington (DC): National Academic Press; 1998.

15. Nusser SM, Carriquiry AL, Dodd KW, Fuller WA. A Semiparametric transformation approach to estimating usual daily intake distributions. J Am Stat Assoc. 1996; 91:1440-9.

16. United States of American. Institute of Medicine. Dietary reference intakes: applications in dietary assessment. Washington (DC): National Academy Press; 2000.

17. Murphy SP, Vorster HH. Methods for using nutrient intake values (NIVs) to assess or plan nutrient intakes. Food Nutr Bull. 2007;28:S51-S60.

18. Santos AL, Leão LSC. Perfil antropométrico de pré-escolares de uma creche em Duque de Caxias, Rio de Janeiro. Rev Paul Med. 2008; 26(3):218-4. doi: 10.1590/S0103-05822008000300004.

19. Silva MV, Ometto $A M H$, Furtuoso MCO, Pipitone MAP, Sturion GL. Acesso à creche e estado nutricional das crianças brasileiras: diferenças regionais, por faixa etária e classes de renda. Rev Nutr. 2000; 13(3):193-9. doi: 10.1590/S1415-527 32000000300006.

20. Fisberg RM, Marchioni DM, Cardoso MR. Estado nutricional e fatores associados ao déficit de crescimento de crianças freqüentadoras de creches públicas do Município de São Paulo, Brasil. Cad Saúde Pública. 2004; 20(3):812-7. doi: 10.1590/S0 102-311X2004000300018.

21. Fernandes TFS, Diniz AS, Cabral PC, Oliveira RJ, Lola MMF, Silva SMM, et al. Hipovitaminose A em pré-escolares de creches públicas do Recife: indicadores bioquímico e dietético. Rev Nutr. 2005; 18(4):471-480. doi: 10.1590/\$1415-52732005000 400003.
22. Castro NJF, Novaes JF, Silva MR, Costa NMB, Franceschini SCC, Tinoco ALA, et al. Caracterização do consumo alimentar, ambiente socioeconômico e estado nutricional de pré-escolares de creches municipais. Rev Nutr. 2005; 18(3):321-30. doi: 10.1590/S1415-52732005000300004.

23. Leite TA, Paula MS, Ribeiro RA, Leite ICG. Cárie dental e consumo de açúcar em crianças assistidas por creche pública. Rev Odont Univ. 1999; 13(1): 13-8. doi: 10.1590/S0103-06631999000100005.

24. Barbosa RM, Soares EA, Lanzillotti HS. Avaliação da ingestão de nutrientes de crianças de uma creche filantrópica: aplicação do consumo dietético de referência. Rev Bras Saúde Matern Infant. 2007; 2:159-66. doi: 10.1590/S1519-3829200700020 0006.

25. Biscegli TS, Corrêa CE, Romera J, Hernandez JL. Avaliação do estado nutricional e prevalência de carência de ferro em crianças freqüentadoras de uma creche. Rev Paul Pediatr. 2007; 24:323-9. doi: 10.1590/S0103-05822007000400007.

26. Rebolla RA, Nardo JR. Estado nutricional de crianças a partir de levantamento em escolas particulares de Maringá. Arq Apadec. 2004; 8:1015-21.

27. Maranhão DG, Sarti CA. Creche e família: uma parceria necessária. Cad Pesqui. 2008; 38(133):171-94. doi: 10.1590/S0100-15742008000100008.

28. Crepinsek MK, Gordon AR, McKinney PM, Condon EEM, Wilson A. Meals offered and served in US public schools: do they meet nutrient standards? JADA. 2009; 109:S31-S43. doi: 10.1016/j.jada.20 08.10.061.

29. Spinelli MGN, Goulart RMM, Santos ALP, Gumiero LDC, Farhud CC, Freitas EV, et al. Consumo alimentar de crianças de 6 a 18 meses em creches. Rev Nutr. 2003; 16(4):409-14. doi: 10.1590/S1415-5 2732003000400004.

30. Briefel RR, Wildon A, Gleason PM. Consumption of low-nutrient, energy-densefoods and beverages at school, home, and other locations among school, home and other locations among school lunch participants and nonparticipants. JADA. 2009; 109:S79-S90. doi: 10.1016/j.jada.2008.10.064.

Recebido em: 28/8/2009

Versão final reapresentada em: 22/2/2010 Aprovado em: 31/5/2010 
